\title{
The Use of 5-Aminosalicylic Acid in Children and Adolescents With Inflammatory Bowel Disease
}

\author{
Christiane Sokollik, MD,* Nicolas Fournier, PhD, $\dagger$ David Rizzuti, MSc,* \\ Christian P. Braegger, MD, PhD, + Andreas Nydegger, MD, PhD, $\S$ \\ Susanne Schibli, MD,* Johannes Spalinger, MD,*\| \\ and the Swiss IBD Cohort Study Group
}

\begin{abstract}
Background: In ulcerative colitis (UC) 5-aminosalicylic acid (5-ASA) is recommended as primary therapy for mild to moderate disease. Topical 5-ASA has been proven especially effective. In Crohn's disease (CD) the evidence for a beneficial role of 5-ASA is weak. We investigated the use of topical and systemic 5-ASA therapy in children and adolescents with inflammatory bowel disease.
\end{abstract}

Materials and Methods: Data of patients younger than 18 years, registered between April 2008 and December 2015 in the Swiss Inflammatory Bowel Disease Cohort, were analyzed.

Results: Three hundred twenty pediatric inflammatory bowel disease patients were included; 189 with CD and 131 with UC. Over one third of UC patients [51 (39\%)] received topical 5-ASA therapy and $43(33 \%)$ received combination therapy during their disease course. UC patients with left-sided colitis or proctitis were more likely to receive topical or combination therapy as compared with patients with pancolitis $(P<0.001$ and $<0.001$, respectively). An increase in the use of topical 5-ASA therapy in UC patients was noted over time from $5 \%$ to $38 \%$. Forty-seven percent of $\mathrm{CD}$ patients were treated with oral 5-ASA during their disease course. The usage was stable over time at approximately $15 \%$ to $20 \%$.

Conclusions: In recent years a very positive trend showing an increase in topical 5-ASA therapy in children and adolescents with UC has been observed. However topical therapy is still used with relative low frequency, especially in patients with a more extensive disease. Conversely, despite weak evidence supporting 5-ASA use in CD patients it has been frequently prescribed. Physicians should continue to encourage their UC patients to use topical therapy.

Key Words: ulcerative colitis, Crohn's disease, 5-ASA, topical therapy, treatment

(J Clin Gastroenterol 2017;00:000-000)

nflammatory bowel disease (IBD) is a chronic disease with recurrent episodes of active illness punctuated by periods of remission. There are 2 major forms of IBD:

Received for publication September 23, 2016; accepted May 10, 2017. From the *Division of Pediatric Gastroenterology, Hepatology and Nutrition, Children's Hospital, University of Bern, Bern; $†$ Institute of Social and Preventive Medicine (IUMSP), University Hospital of Lausanne; §Division of Pediatric Gastroenterology and Hepatology, University Hospital of Lausanne, Lausanne; $₫$ Division of Pediatric Gastroenterology and Nutrition and Children's Research Center, University Children's Hospital of Zurich, Zurich; and $\|$ Division of Pediatric Gastroenterology, Children's Hospital of Lucerne, Lucerne, Switzerland

The authors declare that they have nothing to disclose.

Address correspondence to: Christiane Sokollik, MD, Division of Pediatric Gastroenterology, Hepatology and Nutrition, Children's Hospital, University of Bern, Freiburgstrasse, 3010 Bern, Switzerland (e-mail: christiane.sokollik@insel.ch).

Copyright (C) 2017 Wolters Kluwer Health, Inc. All rights reserved.

DOI: 10.1097/MCG.0000000000000864 ulcerative colitis (UC) and Crohn's disease (CD). The 2 subtypes differ in symptomology and localization patterns. This explains overlapping treatment strategies yet differing efficacy of medications depending on the subtype. ${ }^{1,2}$

For mild to moderate UC 5-aminosalicylic acid (5-ASA) is the primary treatment choice for induction and maintenance of remission in pediatric and adult patients. ${ }^{2,3}$ Topical 5-ASA has been proven effective especially in distal disease in both children and adults. ${ }^{4}$ Several studies have shown combination therapy of oral and topical 5-ASA to be more effective than monotherapy, even in extensive disease. ${ }^{5,6}$ This is particularly important in pediatric patients, who present with extensive disease more often than adults. ${ }^{7}$

Topical steroid preparations have also been used to treat UC, although they are slightly inferior to topical 5-ASA in inducing remission. ${ }^{8}$ They provide an alternative treatment option for patients who are intolerant to 5-ASA. ${ }^{9}$ Importantly the combination of topical 5-ASA and topical steroids seems to be superior to a single-agent therapy. ${ }^{10}$

The value of 5-ASA in CD therapy is controversial. ${ }^{11}$ Despite the controversy and lack of evidence for therapeutic benefit many CD patients undergo 5-ASA treatment during their disease course. ${ }^{12}$ The likelihood of having used 5-ASA appears to be associated with longer disease duration and colonic location at CD diagnosis. ${ }^{12}$ There are no data available about topical 5-ASA therapy in colonic CD.

On the basis of the currently available data, topical therapy with its favorable risk-benefit profile of good efficacy and low rate of side effects should be employed whenever possible in UC patients. Unfortunately, data from adult patients has demonstrated that the frequency of topical therapy is relatively low, even in patients with proctitis and left-sided colitis. ${ }^{13}$

The aim of this study was to investigate the use of topical and systemic 5-ASA therapies in children and adolescents with inflammatory bowel disease in the Swiss IBD cohort. We hypothesized that in pediatric UC patients topical therapy is used more often after the publishing of new treatment guidelines. ${ }^{2} \mathrm{We}$ also asked whether there is a disease location dependency for 5-ASA use in UC patients and examined the evolution of oral 5-ASA use in CD patients over time.

\section{MATERIALS AND METHODS}

Since the end of 2006 the Swiss IBD Cohort Study (SIBDCS) has been retrospectively and prospectively collecting data on adult IBD patients in Switzerland. ${ }^{14}$ In addition, data on pediatric patients have been collected by 
the SIBDCS since 2008. Patients are recruited by pediatric gastroenterologists during regular follow-up. Owing to the structure of the health care system in Switzerland nearly all pediatric gastroenterologists are hospital-based and the majority of pediatric IBD patients are followed in pediatric academic health centers or specialized clinics. Inclusion criteria are an established IBD diagnosis at least 4 months before enrollment and a diagnosis based on Lennard-Jones diagnostic criteria, confirmed by radiology, endoscopy, or surgery. ${ }^{15}$ The study is approved by local ethic committees, and informed consent was obtained from patients and/or their caregivers before inclusion in the SIBDCS.

Our data analysis included pediatric and adolescent IBD patients under the age of 18. SIBDCS data regarding diagnosis, age at diagnosis, gender, weight, height at diagnosis, initial disease location, and medication history were retrieved. Patients with a change of diagnosis during follow-up and IBD unclassified were excluded from analysis.

\section{Statistics}

Data were analyzed with Stata (v14.1; StataCorp, College Station, TX). Continuous data distribution was assessed using normal QQ plots. Distribution of continuous Gaussian data was summarized as mean $\pm \mathrm{SD}$ and range, whereas distribution of non-Gaussian data was presented as median, interquartile range, and range. Differences in Gaussian-distributed data means between 2 groups were assessed using the Student $t$ test, and in nonGaussian data by using the Wilcoxon Mann-Whitney rank-sum test.

Categorical data were summarized as frequency and group percentages. The $\chi^{2}$ test or the Fisher exact test in cases with insufficient sample size was used for differences in categorical data distribution across groups, for paired nominal data McNemar test was utilized. The Bonferroni correction was applied in cases with multiple testing. Multilevel mixed-effect logistic modeling was used to assess the effect of covariates on binary outcomes when dealing with repeated measurements. A $P$-value $<0.05$ was considered as statistically significant.

\section{RESULTS}

\section{Baseline Characteristics}

In total, 354 pediatric and adolescent patients were enrolled in the SIBDCS between April 2008 and December 2015. After excluding 11 patients as they changed diagnosis during follow-up and 23 patients because they were diagnosed with IBD unclassified, 320 patients were included in the analysis (Table 1). Gender distribution differed in CD with more males diagnosed, whereas in UC gender distribution was nearly equal. There was no difference in age at diagnosis between $\mathrm{CD}$ and $\mathrm{UC}(P=0.091)$; however, CD patients weighed significantly less $(P=0.015)$ and had a lower body mass index $(P=0.006)$.

A first year follow-up was completed for $223(69.7 \%)$ patients, whereas the remaining $97(30.3 \%)$ had only a documented enrollment visit. Furthermore in $82(25.6 \%)$ patients 3 or more yearly follow-ups were available for analysis.

Analyzing disease location at diagnosis revealed that most UC patients presented with extensive colitis or pancolitis [88 (67\%)] and only $24(18 \%)$ with left-sided colitis or proctitis [13 $(10 \%)$ ] (Table 2$)$. Most CD patients had ileocolonic disease, whereas a minority presented with ilealonly or colonic-only disease [according to Paris classification: L1 (ileal) 24 (13\%), L2 (colonic) 24 (13\%), L3 (ileocolonic) $127(67 \%)$, L4 (upper only) 3 (2\%)]. Disease location was not stated in $11(6 \%) \mathrm{CD}$ patients and $6(5 \%)$ UC patients.

\section{Oral and Topical 5-ASA and Topical Steroids}

Almost $40 \%$ of UC patients were ever treated with topical 5-ASA. UC patients with left-sided colitis or proctitis were more likely to receive topical or combination therapy as compared with patients with pancolitis $(P<0.001$ and $<0.001$, respectively) (Table 2$)$. Topical steroid therapy was used to treat UC patients less frequently than topical 5 -ASA $(15 \%$ vs. $39 \%$, respectively, $P<0.001)$. A minority of patients received both topical 5ASA and topical steroids.

For CD, 13 patients were treated with topical 5-ASA; all presented with colonic or ileocolonic disease at diagnosis.

TABLE 1. Baseline Characteristics of Study Population

\begin{tabular}{|c|c|c|c|c|}
\hline Characteristics & $\begin{array}{c}\text { All IBD Patients } \\
\text { (320) }\end{array}$ & $\begin{array}{c}\text { CD Patients } \\
\text { [189 (59.1\%)] }\end{array}$ & $\begin{array}{c}\text { UC Patients } \\
\text { [131 }(40.9 \%)]\end{array}$ & $P, \mathrm{CD}$ vs. $\mathrm{UC}$ \\
\hline \multicolumn{5}{|l|}{ Gender } \\
\hline Male & $174(54.4)$ & $109(57.7)$ & $65(49.6)$ & 0.155 \\
\hline Female & $146(45.6)$ & $80(42.3)$ & $66(50.4)$ & \\
\hline Age at diagnosis (y) (median, IQR) & $12,9-14$ & $12,10-14$ & $12,9-14$ & 0.091 \\
\hline Age at enrollment (y) (median, IQR) & $14,12-16$ & $14,12-16$ & $14,12-16$ & 0.243 \\
\hline BMI $z$ score at enrollment (mean, SD) & $-0.28,1.06$ & $-0.42,1.09$ & $-0.08,0.98$ & 0.006 \\
\hline Height $z$ score at enrollment (mean, SD) & $-0.28,2.11$ & $-0.41,1.94$ & $-0.09,2.33$ & 0.189 \\
\hline Weight $z$ score at enrollment (mean, SD) & $-0.40,1.17$ & $-0.57,1.16$ & $-0.16,1.17$ & 0.015 \\
\hline Age at latest FUP (y) (median, IQR) & $16,14-17$ & $17,14-17$ & $16,13-17$ & 0.141 \\
\hline Disease duration (y) (median, IQR)* & $3,2-5$ & $3,2-5$ & $3,1-6$ & 0.757 \\
\hline \multicolumn{5}{|l|}{ Follow-up [n (\%)] } \\
\hline Enrollment only & $97(30.3)$ & $54(28.6)$ & $43(32.8)$ & \\
\hline 1st year FUP & $83(25.9)$ & $47(24.9)$ & $36(27.5)$ & \\
\hline 2nd year FUP & $58(18.1)$ & $39(20.6)$ & $19(14.5)$ & \\
\hline 3rd year FUP or more & $82(25.6)$ & $49(25.9)$ & $33(25.2)$ & 0.515 \\
\hline
\end{tabular}

*Disease duration $=$ age at last follow-up -age at diagnosis.

BMI indicates body mass index; CD, Crohn's disease; FUP, follow-up; IBD, inflammatory bowel disease; IQR, interquartile range; UC, ulcerative colitis. 


\begin{tabular}{lccccc}
\hline \multicolumn{7}{l}{ TABLE 2. 5-ASA and Steroid Therapy in UC Patients Depending on Disease Location } & & \\
\hline Medication & $\begin{array}{r}\text { Pancolitis } \\
{[\mathbf{8 8}(\mathbf{7 0 . 4 \% )}]}\end{array}$ & $\begin{array}{c}\text { Left-sided } \\
{[\mathbf{2 4}(\mathbf{1 9 . 2 \% )}]}\end{array}$ & $\begin{array}{c}\text { Proctitis } \\
{[\mathbf{1 3}(\mathbf{1 0 . 4} \%)]}\end{array}$ & $\begin{array}{c}\text { Overall } \\
\boldsymbol{P}\end{array}$ & $\begin{array}{c}\boldsymbol{P} \text {, Pancolitis vs. Left-sided } \\
\text { Plus Proctitis }\end{array}$ \\
\hline Oral 5-ASA & $83(94.3)$ & $24(100.0)$ & $10(76.9)$ & 0.032 & 0.613 \\
Topical 5-ASA & $26(29.5)$ & $14(58.3)$ & $11(84.6)$ & $<0.001$ & $<0.001$ \\
Oral + top 5-ASA & $21(23.9)$ & $14(58.3)$ & $8(61.5)$ & 0.001 & 0.001 \\
Systemic steroids & $69(78.4)$ & $16(66.7)$ & $6(46.2)$ & 0.038 & 0.030 \\
Topical steroids & $10(11.4)$ & $5(20.8)$ & $3(23.1)$ & 0.280 & 0.136 \\
\hline
\end{tabular}

Six UC patients were excluded from analysis due to unknown disease location at diagnosis.

5-ASA indicates 5-aminosalicylic acid; UC, ulcerative colitis.

Nearly $50 \%$ of CD patients received oral 5-ASA during their disease course (Table 3).

Combination therapy of topical and oral 5-ASA was given to $32.8 \%$ of UC patients and $3 \%$ of $\mathrm{CD}$ patients $(P<0.001)$.

The use of topical 5-ASA was found to be independent of disease duration [median disease duration (interquartile range, range) users versus nonusers, 3 y ( 1 to 6,0 to 9 ) and 3 y (2 to 5,0 to 16$)$, respectively, $P=0.558$ ] and age of diagnosis [11 y (9 to 13,3 to 17$)$ and 12 y (10 to 14,0 to 17$)$, respectively, $P=0.059]$. Topical 5-ASA users were more likely to be female than male, although this difference was not statistically significant [36 (56.3\%) and $28(43.7 \%)$, respectively, $P=0.059$ ].

Analysis of the usage per year revealed an increase in the use of topical 5-ASA therapy in UC patients over time (from $<5 \%$ in 2009 to $>38 \%$ in 2015) (Fig. 1A). This increase in percentage treated with topical 5-ASA was statistically significant in 2014 and 2015 with respect to previous years $(P=0.022)$. In addition, for the years 2013, 2014 , and 2015, increases in the continuous use of topical 5ASA could be documented of $5.1 \%, 7.3 \%$, and $12.8 \%$, respectively. In CD patients the usage of oral 5-ASA was stable over time as each year $15 \%$ to $20 \%$ of CD patients received oral 5-ASA (Fig. 1B).

\section{Other Medications}

There was no difference in the use of systemic steroid therapy between UC and CD patients $(P=0.527)$ (Table 3$)$. Budesonide was used to treat $\mathrm{CD}$ patients significantly more often than UC patients $(P<0.001)$. Significantly more CD than UC patients received anti-tumor necrosis factor- $\alpha$ antibody therapy $(P<0.001)$. CD patients were also treated more often with immunomodulators and antibiotics than UC patients $(P<0.001$ and 0.018 , respectively).

\section{DISCUSSION}

In this study we described the treatment modalities of children and adolescents with inflammatory bowel diseases and focused on 5-ASA therapy.

As expected and in concordance with actual guidelines ${ }^{1,2}$ treatment strategies differed between patients with $\mathrm{CD}$ and UC. Patients with $\mathrm{CD}$ received systemic therapy with budesonide, anti-tumor necrosis factor- $\alpha$ antibodies, immunomodulators, and antibiotics significantly more often than UC patients. Oral and topical 5-ASA was used significantly more often as a treatment in UC patients.

\section{5-ASA in UC}

As previously described for other pediatric cohorts ${ }^{7}$ most of our pediatric and adolescent UC patients already presented with extensive disease at diagnosis and only a minority with proctitis. Topical 5-ASA was shown to be effective in children with proctitis ${ }^{4}$ and the majority of patients in our cohort with proctitis received topical 5-ASA. As combining oral 5-ASA with topical 5-ASA is more effective than oral alone, combination therapy should be offered for induction of remission for mild to moderate disease even in extensive disease according to the actual evidence-based treatment guideline. ${ }^{2}$ In our cohort only $38.9 \%$ of UC patients had ever been treated with topical 5ASA and $32.8 \%$ with combination therapy of oral and topical 5-ASA. Despite these relatively low numbers, there was a very gratifying trend in our cohort of increased prescription of topical 5-ASA in the years subsequent to publication of the new treatment guidelines in $2012 .^{2}$ In addition, more patients in our study were treated with

TABLE 3. Medication Use in General

\begin{tabular}{|c|c|c|c|c|}
\hline Medication & All IBD Patients (320) & CD Patients [189 (59.1\%)] & UC Patients [131 (40.9\%)] & $P, \mathrm{CD}$ vs. $\mathrm{UC}$ \\
\hline Oral 5-ASA & $211(65.9)$ & $89(47.1)$ & $122(93.1)$ & $<0.001$ \\
\hline Topical 5-ASA & $64(20.0)$ & $13(6.9)$ & $51(38.9)$ & $<0.001$ \\
\hline Oral + top 5-ASA & $49(15.3)$ & $6(3.1)$ & $43(32.8)$ & $<0.001$ \\
\hline Systemic steroids & $238(74.4)$ & $143(75.7)$ & $95(72.5)$ & 0.527 \\
\hline Budesonide & $46(14.4)$ & $40(21.2)$ & $6(4.6)$ & $<0.001$ \\
\hline Top steroids & $33(10.3)$ & $14(7.4)$ & $19(14.5)$ & 0.040 \\
\hline Top 5-ASA + top steroids & $13(4.1)$ & $4(2.1)$ & $9(6.9)$ & 0.044 \\
\hline Antibiotics & $107(33.4)$ & $73(38.6)$ & $34(26.0)$ & 0.018 \\
\hline Immunomodulators & $253(79.1)$ & $169(89.4)$ & $84(64.1)$ & $<0.001$ \\
\hline anti-TNF- $\alpha$ antibody & $139(43.4)$ & $104(55.0)$ & $35(26.7)$ & $<0.001$ \\
\hline
\end{tabular}

5-ASA indicates 5-aminosalicylic acid; CD, Crohn's disease; IBD, inflammatory bowel disease; TNF- $\alpha$, tumor necrosis factor- $\alpha$; top, topical; UC, ulcerative colitis. 


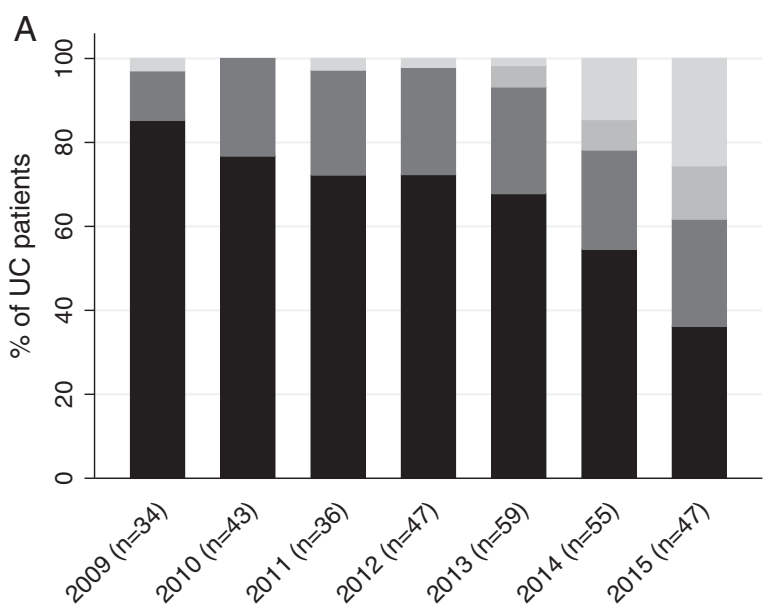

Topical 5-ASA usage

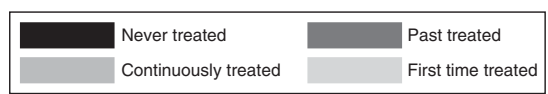

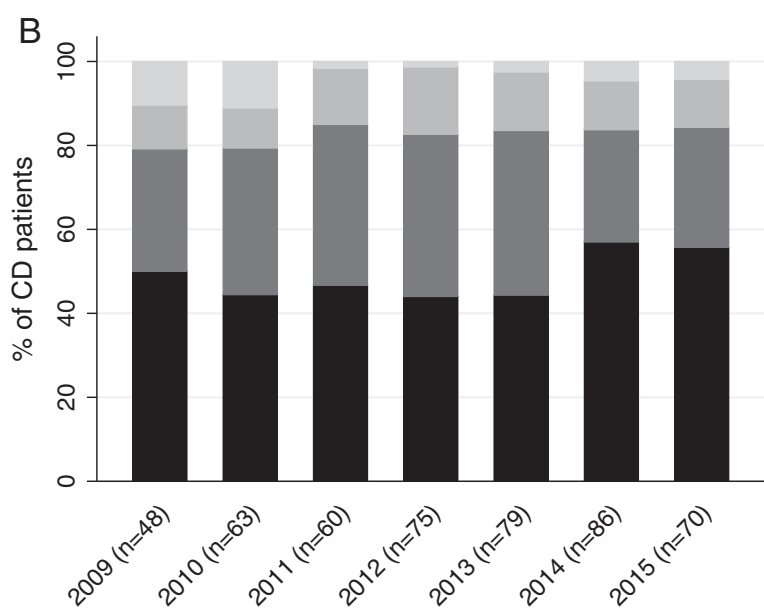

Oral 5-ASA usage

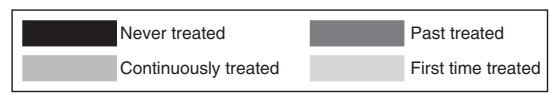

FIGURE 1. Change of 5-aminosalicylic acid (5-ASA) use over time. A, Analysis of the 5-ASA usage per year from 2009 to 2015 revealed a gradual increase in the use of topical 5-ASA therapy in ulcerative colitis (UC) patients. B, Oral 5-ASA use in Crohn's disease (CD) patients was stable over the same time period. The number in parentheses indicates the number of patients diagnosed in the given year. First time treated, inclusion of 5-ASA into therapy; continuously treated, 2 or more consecutive years of 5-ASA treatment including the given year.

topical therapy as compared with a similar study in adults, in which only $17.7 \%$ of a cohort of 773 UC patients had ever received topical 5-ASA therapy and only $9.3 \%$ a 5 ASA combination therapy. ${ }^{13}$

There are diverse explanations for the low frequency of topical therapy usage. Physician's reservations for prescribing topical therapies due to assumed inconvenience for the patients may be one factor. Another factor could be difficulties in following a daily dosage regimen due to school and sport commitments. A busy life style was one of the most common reasons reported by adult patients for intentional nonadherence to rectal therapy in an observational study. ${ }^{16}$ However, frequency may be tailored to individual patient needs. In a randomized controlled trial involving UC patients in remission weekend application of 5-ASA enema in combination with oral 5-ASA significantly reduced the incidence of relapses. ${ }^{17}$ This indicates that a once-daily administration is not always required and a less frequent schedule adjusted to the patients' schedule may be also effective.

Studies examining health-related quality of life actually showed an improvement in patients on combination therapy. 6,18 This increase in quality of life was attributed to significant differences in mobility, usual care, and anxiety/depression. One contributing factor may be the shorter time to bleeding cessation documented with the use of topical therapy. ${ }^{5,6}$ Interestingly, when queried on future usage, the majority of patients saw themselves prepared to use combination therapy again in the future. ${ }^{6}$ Thus combination therapy should be reoffered during the disease course.

Different 5-ASA formulations, including foam and liquid enema, are available with comparable efficacy. ${ }^{19}$ Foam retention was shown to be significantly better than liquid retention and may be the preferred formulation in patients with a severely inflamed rectum. ${ }^{19}$ Topical therapy and the different formulations should be explained to patients and explored whenever possible.

\section{5-ASA in CD}

Previously it was reported that 5-ASA is frequently prescribed in adult $C D$ patients ${ }^{12}$ despite lack of scientific support for a beneficial role. ${ }^{20}$ Furthermore nearly half of the CD patients in our cohort were treated with oral 5-ASA during their disease course. Earlier pediatric studies which suggested a possible benefit of 5-ASA in the treatment of small intestinal $C^{21}$ may have influenced these prescribing practices. Consequentially 5-ASA was considered a valuable treatment option in mild to moderate pediatric $\mathrm{CD}$ in previous consensus summaries. ${ }^{22}$ However a recent metaanalysis has reaffirmed that 5-ASA has little benefit for the treatment of active CD. ${ }^{20}$ In concordance with this evidence the actual treatment guideline for pediatric CD recommends oral 5-ASA only for selected patients with a very mild colonic disease. ${ }^{1}$ Only $13 \%$ of $\mathrm{CD}$ patients in our cohort had colonic-only disease at diagnosis, whereas nearly half of all CD patients received oral 5-ASA at some time point. The yearly prescription rate of 5-ASA in CD patients was stable between 2009 and 2015; however, the effect and the adjustment of treatment modalities from the 2014 published pediatric guideline may not yet be evident. A recent study from Israel comparing the intent to use specific treatments in adult and pediatric IBD patients showed that $60 \%$ of pediatric gastroenterologists would use 5 -ASA in mild CD and $21 \%$ in Crohn's colitis, ${ }^{23}$ indicating that 5-ASA use in pediatric CD is still common and not specific for Switzerland.

Interestingly, 1 study in adults demonstrated that physicians perceived a clinical response to 5-ASA in nearly half of their CD patients. ${ }^{12}$ Whether this represents a true drug effect or simply a biased patient selection with a priori benign natural course of $\mathrm{CD}$ is debatable, as it is known 
that Crohn's colitis can remain uncomplicated for many years in the adult population. ${ }^{24}$ In pediatric CD the disease course in general is less benign. However, whether there exists a similar perception among pediatric gastroenterologists and the extent to which the adult perception influences the pediatric one remains speculative.

Another pertinent factor for 5-ASA prescription in $\mathrm{CD}$ especially in pediatrics may be that 5-ASA is regarded as a mild and safe medication with a low side effect rate, reducing the threshold for prescription. However, there is a potential for severe side effects ${ }^{25}$ and therefore all prescriptions should be carefully evaluated to appropriately weigh risk and benefits.

\section{Strength and Limitations}

A major strength of this study is the size of the cohort with coverage of the majority of pediatric and adolescents IBD patients in Switzerland. These patients are almost universally cared for at pediatric academic health centers or specialized clinics in Switzerland which are part of the SIBDCS. Another strength is the covered time period of 7 years with a systematic recording of patients' data. This allowed us to determine some trends in treatment modalities. The main limitation of our study is the retrospective observational design in which database records are analyzed. This design does not enable assessment of adherence to the prescribed medication or clinical response. However, the documented increase in the number of continuously topical 5-ASA treated patients may indicate good compliance.

\section{CONCLUSIONS}

Topical therapy is still underused in children and adolescent with UC, although a very positive trend of increased prescription and continuously use could be noted. Physicians should continue to encourage and educate their patients to improve adherence to and compliance with a therapy which may initially appear very unpleasant. Optimized utilization of different frequency regimens and drug formulations may further promote compliance and adherence.

In contrast oral 5-ASA is still used frequently in pediatric and adolescent CD patients despite a lack of strong supporting evidence. This gap between scientific evidence and clinical practice may close with the further spread of guidelines.

\section{REFERENCES}

1. Ruemmele FM, Veres G, Kolho KL, et al. Consensus guidelines of ECCO/ESPGHAN on the medical management of pediatric Crohn's disease. J Crohns Colitis. 2014;8:1179-1207.

2. Turner D, Levine A, Escher JC, et al. Management of pediatric ulcerative colitis: joint ECCO and ESPGHAN evidence-based consensus guidelines. J Pediatr Gastroenterol Nutr. 2012;55: 340-361.

3. Dignass A, Lindsay JO, Sturm A, et al. Second European evidence-based consensus on the diagnosis and management of ulcerative colitis part 2: current management. J Crohns Colitis. 2012;6:991-1030.

4. Heyman MB, Kierkus J, Spenard J, et al. Efficacy and safety of mesalamine suppositories for treatment of ulcerative proctitis in children and adolescents. Inflamm Bowel Dis. 2010;16: 1931-1939.

5. Marteau P, Probert CS, Lindgren S, et al. Combined oral and enema treatment with Pentasa (mesalazine) is superior to oral therapy alone in patients with extensive mild/moderate active ulcerative colitis: a randomised, double blind, placebo controlled study. Gut. 2005;54:960-965.
6. Probert CS, Dignass AU, Lindgren S, et al. Combined oral and rectal mesalazine for the treatment of mild-to-moderately active ulcerative colitis: rapid symptom resolution and improvements in quality of life. J Crohns Colitis. 2014;8: 200-207.

7. Van Limbergen J, Russell RK, Drummond HE, et al Definition of phenotypic characteristics of childhood-onset inflammatory bowel disease. Gastroenterology. 2008;135: $1114-1122$.

8. Marshall JK, Irvine EJ. Rectal corticosteroids versus alternative treatments in ulcerative colitis: a meta-analysis. Gut. 1997:40:775-781

9. Navarro F, Hanauer SB. Treatment of inflammatory bowel disease: safety and tolerability issues. Am $J$ Gastroenterol. 2003;98:S18-S23.

10. Mulder CJ, Fockens P, Meijer JW, et al. Beclomethasone dipropionate $(3 \mathrm{mg})$ versus 5-aminosalicylic acid $(2 \mathrm{~g})$ versus the combination of both $(3 \mathrm{mg} / 2 \mathrm{~g})$ as retention enemas in active ulcerative proctitis. Eur J Gastroenterol Hepatol. 1996;8: 549-553.

11. Moja L, Danese S, Fiorino G, et al. Systematic review with network meta-analysis: comparative efficacy and safety of budesonide and mesalazine (mesalamine) for Crohn's disease. Aliment Pharmacol Ther. 2015;41:1055-1065.

12. Schoepfer AM, Bortolotti M, Pittet V, et al. The gap between scientific evidence and clinical practice: 5 -aminosalicylates are frequently used for the treatment of Crohn's disease. Aliment Pharmacol Ther. 2014;40:930-937.

13. Seibold F, Fournier N, Beglinger C, et al. Topical therapy is underused in patients with ulcerative colitis. J Crohns Colitis. 2014;8:56-63.

14. Pittet V, Juillerat P, Mottet C, et al. Cohort profile: the Swiss Inflammatory Bowel Disease Cohort Study (SIBDCS). Int $J$ Epidemiol. 2009;38:922-931.

15. Braegger CP, Ballabeni P, Rogler D, et al. Epidemiology of inflammatory bowel disease: is there a shift towards onset at a younger age? J Pediatr Gastroenterol Nutr. 2011;53:141-144.

16. Boyle M, Ting A, Cury DB, et al. Adherence to rectal mesalamine in patients with ulcerative colitis. Inflamm Bowel Dis. 2015;21:2873-2878.

17. Yokoyama H, Takagi S, Kuriyama S, et al. Effect of weekend 5-aminosalicylic acid (mesalazine) enema as maintenance therapy for ulcerative colitis: results from a randomized controlled study. Inflamm Bowel Dis. 2007;13:1115-1120.

18. Connolly MP, Poole CD, Currie CJ, et al. Quality of life improvements attributed to combination therapy with oral and topical mesalazine in mild-to-moderately active ulcerative colitis. Digestion. 2009;80:241-246.

19. Cortot A, Maetz D, Degoutte E, et al. Mesalamine foam enema versus mesalamine liquid enema in active left-sided ulcerative colitis. Am J Gastroenterol. 2008;103:3106-3114.

20. Lim WC, Hanauer S. Aminosalicylates for induction of remission or response in Crohn's disease. Cochrane Database Syst Rev. 2010:CD008870.

21. Griffiths A, Koletzko S, Sylvester F, et al. Slow-release 5aminosalicylic acid therapy in children with small intestinal Crohn's disease. J Pediatr Gastroenterol Nutr. 1993;17: 186-192.

22. Levine A, Milo T, Buller H, et al. Consensus and controversy in the management of pediatric Crohn disease: an international survey. J Pediatr Gastroenterol Nutr. 2003;36:464-469.

23. Assa A, Avni I, Ben-Bassat O, et al. Practice variations in the management of inflammatory bowel disease between pediatric and adult gastroenterologists. J Pediatr Gastroenterol Nutr. 2016;62:372-377.

24. Cosnes J, Gower-Rousseau C, Seksik P, et al. Epidemiology and natural history of inflammatory bowel diseases. Gastroenterology. 2011;140:1785-1794.

25. Kohli R, Melin-Aldana H, Sentongo TA. Mesalamine-induced pneumonitis during therapy for chronic inflammatory bowel disease: a pediatric case report. J Pediatr Gastroenterol Nutr. 2005;41:479-482. 